\title{
El hilo cronológico de Los Bravos de Jesús Fernández
}

\section{Santos}

\section{Heba Mohamed Ibrahim Abdel Latif}

Dpto. de Lengua Española, Facultad de Al-Alsun, Universidad de Minia, Egipto

Email: heba.ibrahim1@mu.edu.eg

Tense is a key component in fiction; no fictional work is void of tense as a narrative element. The narration art developed from a simple form relying on a chronological sequence of events to a more complicated form depending on an overlap of tenses. The present study is aimed at analyzing the narrative tense in Jesús Fernández Santos' first novel The Brave (Los bravos). Santos represents the generation of Spanish novelists in the mid-twentieth century. This topic is important as most events in Los bravos are sequential and follow each other with some narrative exceptions. This is due to the fact that Santos manipulates tenses, starting from present to past, then from near past to distant past, and from present to future. Also, Santos uses the synchronization technique to offer an impression of real life as everyone's life in the village is considered part of the world where events are synchronized.

Keywords (The Brave) Los Bravos, Jesús Fernández Santos, narration, narrative tense, narrative techniques

\section{Resumen}

El tiempo es una categoría muy importante para el género narrativo.

Pues, es imposible que no esté en mayor o menor grado en una narración. 
El tiempo narrativo es un eje transversal a toda narración que determina a los acontecimientos, a la vitalidad de los personajes que avanzan, crecen y envejecen (o al revés, van haciéndose más jóvenes, retrocediendo), al ambiente físico y climático, etc.

Por eso, el objetivo de este trabajo es analizar el tiempo narrativo de Los bravos, la primera novela de Jesús Fernández Santos, uno de los representantes más sobresalientes de la generación del medio siglo español. El estudio del tiempo narrativo de Los bravos adquiere una relevancia especial, pues, el autor crea una indeterminación cronológica junto a una reiteración del espacio narrativo, la cual se debe, finalmente, al deseo de universalizar la obra para cualquier época.

La mayor parte de los hechos de la novela son lineales, con unas alteraciones cronológicas que disponen el autor para dar una mayor estética a la narración.: Analepsis y Prolepsis. También, la técnica de la simultaneidad, utilizada para dar una mayor impresión de la realidad cotidiana. Pues, la vida de cada persona en el pueblo es una parte del mundo en el que suceden acciones simultáneas. Así, Jesús Fernández Santos no realiza sólo una exposición paralela de secuencias, sino que introduce éstas en pleno diálogo para darnos información sobre lo que ocurre en otro lugar o para enfocar otra acción, que sucede en el mismo momento.

Palabras clave: Los bravos, Jesús Fernández Santos, narración, tiempo narrativo, técnicas narrativas

\section{Introducción}

Ya es sabido que el tiempo es una categoría muy importante para el género narrativo. Pues, es imposible que no esté en mayor o menor grado en una narración. En este sentido, algunos críticos han llegada a señalar que el texto narrativo es: "una cronofanía, una modalidad artística en la que el tiempo adquiere una relevancia sustancial" según Villanueva (1989, p. 44).

Se pueden plantear varias interrogaciones sobre el tiempo. Chatman ya 
hace algunas preguntas, en mi opinión, muy acertadas sobre el uso del tiempo:

¿Qué relaciones hay entre el orden natural de los acontecimientos en la historia y el orden de tu presentación para el discurso? ¿Y entre la duración de la presentación discursiva y la de los hechos reales de la historia? ¿Cómo presenta el discurso eventos recurrentes? (1990, p. 66)

Todas estas preguntas se deben a la manipulación del novelista, ya que no en vano, la historia tiene un orden cronológico que corresponde a los hechos ocurridos y cualquier alteración puede atribuirse a la manipulación en el paso de la historia al argumento. Tanto la extensión de cada argumento como la forma de contarlo, omitiendo o alargando, debe analizarse a partir de un punto de vista temporal, por lo que, según Bobes Naves:

el tiempo alcanza gran relieve como elemento organizador de la novela, es decir, en su aspecto sintáctico. En el paso de la historia al argumento, el tiempo es la categoría fundamental para establecer las referencias. (1998, p. 168).

Villanueva mantiene una idea similar sobre este asunto, y sostiene que el tiempo se considera como elemento organizador de la obra literaria. Así, el paso de la historia al argumento, se debe analizar desde un punto de vista temporal.

Analizar este decisivo factor estructurante en un cuento o novela pasa ineludiblemente por el estudio de cómo el tiempo de la historia se ha transformado en el único textualmente pertinente, el tiempo del discurso. (1989, p. 44)

\section{El objetivo del trabajo}

Debido a lo anterior, el objeto de este trabajo es analizar el tiempo narrativo que es un eje transversal a toda narración que determina a los acontecimientos, a la vitalidad de los personajes que avanzan, crecen y envejecen (o al revés, van haciéndose más jóvenes, retrocediendo), al ambiente físico y climático, etc. A través de la novela Los bravos de Jesús Fernández Santos. Por ello, se atiende, de forma somera, a la personalidad, la formación, circunstancias en las cuales se inicia en el quehacer literario y ambientes en los que se mueve el escritor en cuanto que tales factores contribuyen en su particular manera de expresar el carácter de su sociedad.

\section{Jesús Fernández Santos}

El nombre de Jesús Fernández Santos ha pasado a la mayoría de los 
manuales de la Historia de la literatura española del siglo XX como uno de los principales representantes de la tendencia llamada "realismo social" que aparece en España en los comienzos de los cincuenta. Asimismo, su primer fruto literario Los bravos 1954, está considerada por buena parta de la crítica como punto de partida de esta tendencia. Nuestro escritor no sólo escribió novelas, sino también redactó cuentos, ensayos, artículos de crítica cinematográfica y guiones, podemos decir que la única excepción fue la poesía. También, no se puede olvidar su trabajo en el cine como director destacándose con varios cortometrajes y su trabajo en la Televisión española. Todo esto, se pone claro que estamos ante un autor singular, de gran bagaje cultural y de pluma sensible.

Jesús Fernández Santos, nació en Madrid el 9 de noviembre de 1926, en el seno de una familia modesta perteneciente a la clase media burguesa de la época. Su padre procedía de un pequeño pueblo de la montaña leonesa, Cerulleda, que sólo dista de la frontera divisoria con Asturias cuatro o cinco kilómetros y su madre de origen vallisoletano. Cuando tenía diez años, estalló la guerra civil española que duró más de mil días y costó ríos de sangre. Así, pasó la adolescencia y la primera juventud en los difíciles años de la inmediata posguerra. Ante tales circunstancias que tenía que soportar casi toda la sociedad española de aquel entonces, el autor sintió profundamente el sufrimiento de su sociedad y reaccionó ante ello tratando modificarlo por los medios que tenía en su alcance, en este caso, el talento de ficción literaria. Desde las primeras manifestaciones, la obra de Fernández Santos ha ido reflejado unas realidades sociales, culturales y económicas de la sociedad, sobre todo, el mundo rural "porque es en la sociedad rural donde existen las mayores injusticias y desigualdades", según Gil Casado: (1975, p. 217

\section{Argumento de Los Bravos}

Esta es la primera obra de Jesús Fernández Santos, y con ella el autor irrumpió el panorama novelístico de los años cincuenta, y dio a conocer en el mundo literario. En realidad, Los bravos había circulado semanalmente como folletín en la revista Ateneo entre 1952 y 1953, pero no llamó mucha atención. En el momento de su aparición, la novela fue 
aplaudida por la crítica y su autor fue considerado uno de los nuevos valores de la narrativa española de la posguerra. La atención por esta obra se debía a que apuntó hacia nuevas direcciones con ingredientes novedosos: técnica cinematográfica, objetividad, nuevos estilos narrativos y crítica social.

La novela examina la humilde vida de los habitantes de un pueblecito leonés en la frontera de Asturias. A pesar de que no queda ubicado con exacto en el relato, sino, a través de las declaraciones del escritor, sabemos que se trata Ceruelleda, donde procedía el padre del autor y donde vivió Jesús Fernández Santos cierto tiempo, y le conoció palmo a palmo. Se trata de un pequeño pueblo, sin iglesia, sin cura y sin riqueza, doce casas, doce vecinos, una carretera que atraviesa, montañas y un río cercano. En el pueblo se trabaja muy duro y los beneficios son escasos. $\mathrm{El}$ autor nos muestra las vidas de hasta 50 personajes cuyas vidas se ven alteradas por la llegada al pueblo de dos forasteros: un médico joven y un viajante.

Don Prudencio, un anciano que salió bien librado de la guerra civil, es el hombre de mayor prestancia; muchos de los otros habitantes han pedido dinero prestado a él para sostener sus cosechas y han terminado endeudándose, por eso lo odian. También porque vive con una muchacha, Socorro, muchísima más joven que él. Vive tan bien como Pilar, la gorda inútil y usurera que manda a traer de afuera los mejores vinos. Los demás, apenas son hombres repetidos, se ganan la vida trabajando de lunes a domingo, cultivan la tierra o crían ganado, pero también pescan en sitios prohibidos, llevan contrabando, o viven con la expectativa de escaparse a la ciudad. Por eso es tan importante para ellos la noche: es el espacio propicio para acechar el río y pescar mientras el guardia está del otro lado, o para que los asturianos vengan desde más arriba para comprar víveres que vienen ilegalmente desde el puerto. Las mujeres están a la sombra de sus hombres, desde niñas, se les había enseñado que habían venido al mundo para servirlos. Así lo hace la esposa de Antón, el secretario del ayuntamiento; las hijas de Alfredo, el campesino amante de las truchas; Socorro, la joven que vive con Don Prudencio y que luego 
pasará a ser amante del médico; la criada de Pilar; la Esposa de Manolo, el de la fonda, etcétera. Sorprende escuchar algunos de sus nombres que por ellos mismos viene a expresar la condición de quienes los llevan: Blanca, Amparo, Socorro, Consuelo o Asunción.

Nada parece alterar esa monotonía: los hombres beben algún trago en la fonda, discuten, hablan de los otros, de lo que les hace falta, de su niñez y tantas otras cosas. Las mujeres preparan el pan, cuidan los cultivos y crían. Los muchachos andan por allí despabilándose en el río o jugando entre los árboles. Sin embargo, ha llegado un médico desde la ciudad que ha venido a reemplazar a don Julián, quien se aburrió muy pronto del pueblo, y el nuevo viene a confundir a los habitantes con las cosas que hace: se enamora de la amante de don Prudencio y se la lleva a vivir a su casa de alquiler, cosa que luego tendrá para el viejo terribles consecuencias; y luego, defiende al viajante que estuvo por el pueblo unos días antes y que, engañando a los campesinos con una supuesta póliza de banco que les aseguraba una vejez tranquila, se había llevado sus ahorros, sólo que no contó con que en otro pueblo lo descubrirían y golpearían, pero no así cuando el médico lo llevó a su casa para salvarlo de la tronera que le esperaba frente a esos bravos que querían resarcir su orgullo.

Nada más. Antonio se casa y es necesario llamar al cura del otro pueblo; arreglan un poco la iglesia abandonada. Un pastor se enferma en las montañas y el médico tiene que subir, mientras escucha de su acompañante cómo hasta hace algunos años en todas esas laderas se seguían encontrando cadáveres. Don Prudencio viaja a la ciudad porque está muy enfermo, pero su hermano ha marchado y, la ciudad, si bien es grande y puede divertirlo, lo aburre. Pepe va y viene en su viejo carro trayendo las diligencias al pueblo desde la estación del tren; por las noches corteja a una de las hijas de don Alfredo, al que le dieron un balazo en la pierna por pescar de noche. Los hombres se lamentan de sus cosechas y azuzan a los más jóvenes para que se larguen pronto. Pepe se va definitivamente; se queda el médico; y también muere alguien con el que se empieza a borrar de la memoria cierto momento de la historia del 
pueblo.

\section{La cronología de Los Bravos de Jesús Fernández Santos}

Por lo que se refiere al tiempo narrativo de la novela social del medio siglo, S. Villanueva apunta que normalmente estas novelas sociales suelen ser historias lineales, con reducción tiempo-espacio, completadas, si es necesario, con breves retrocesos. En cuanto a esta reducción mencionada, se puede señalar la relación de este rasgo con la presencia del protagonismo colectivo y típico de la narrativa de los años cincuenta para lograr un efecto de condensación (1980, pp. 205-207).

En este punto, Sobejano subraya la tendencia objetivista de la generación del medio siglo en librarse del predominio del autor, y destaca el uso de los monólogos para romper la continuidad y mostrar el tiempo interno; valora, de manera especial, la técnica cinematográfica: los cortes espaciales, los enfoques y valores visuales, los fundidos, disolvencias, retrocesos e imágenes superpuestas, $\mathrm{y}$, en fin, el montaje para mostrar la multiplicidad y superar los límites espaciotemporales. Por influencia del cine, Sobejano destaca la temporalización del espacio y la especialización del tiempo (1975, pp. 361-363).

En el caso de Jesús Fernández Santos, el estudio del tiempo narrativo de su obra adquiere una relevancia especial, pues, el autor crea una indeterminación cronológica junto a una reiteración del espacio narrativo. Esta imprecisión temporal aparece en Los bravos y más en la mayoría de su producción literaria, la cual se debe, finalmente, al deseo de universalizar su obra para cualquier época. Así, el lector percibe la duración temporal. Al respecto J. Madrid opina que:

En Los bravos, y con posterioridad a toda su producción, el escritor madrileño se mueve dentro de unos mecanismos genéricos, globalizadores, vagos, descuidando la precisión y la exactitud cronológica y temporal. El autor, por deseos de universalizar, elige una época que puede ser cualquiera o, por la utilización del presente, puede resultar válido por todas las épocas. (1991, p. 41)

Generalmente, el tiempo tratado en Los bravos se considera un tiempo lineal y cronológico, sin grandes modificaciones. Sin embargo, hay algunos retrocesos y varios momentos de simultaneidad, lo cual 
constituye una de las técnicas más utilizadas en la narración del medio siglo y una característica constante en la obra fernándezsantiana, como vamos a analizar en las líneas siguientes.

\section{Tiempo lineal y cronológico}

En cuanto a Los bravos, la mayor parte de las cuarenta y dos secuencias en las que consiste la novela, presenta una temporalidad lineal y progresiva. Los críticos anteriores a J. Madrid señalaron la imprecisión temporal de la narración, pero mencionaron que las acciones transcurren durante un período de verano caluroso sin precisar los días pasados. Gil Casado señala que la acción de la novela ocurre "durante un caluroso verano" (1975, p. 197). También, Eugenio de Nora declara que la narración transcurre "a lo largo de un verano" (1937, p. 289). J. Madrid ha sido más preciso en su estudio de la estructura secuencial de la novela y determina que sus acciones duran catorce días, gracias a las referencias implícitas y explícitas que el mismo Jesús Fernández Santos ha mencionado en el texto, que sirven al lector para conocer el avance del tiempo y la época en la que se encuentra.

Igualmente, al estudiar el tratamiento temporal de Los bravos debemos aludir a dos referencias temporales muy importantes, la primera, nos informa que el transcurso de la narración se sitúa en el mes de agosto, ya que así se menciona en el diálogo mantenido entre la madre de Amador y el estafador:

- ¿Le molestan las moscas?

-Todavía no.

-Si estuviera para el mes que viene, ya vería. Septiembre es el mes de las moscas. -Hizo una pausa, y como el viajante no contestaba, preguntó- ¿Me oye? (Los bravos, pp. 74-75).

En la conversación que mantiene Antonio, el herrero, con unos guardias, tenemos la segunda referencia que nos indica que el tercer día es un sábado, con lo cual supone que el primer día es un jueves, como hemos aludido antes, según la cita mencionada de la novela: (Los bravos, p. 88) partiendo del deseo de no repetirla.

El cambio del día en la novela depende de varias ocasiones; de agentes naturales como el sol, la luna, las estrellas, el alba y, desde luego, las 
marcas del reloj o, como observó J. Madrid, “por acciones que ejecutan los personajes como apagar el fuego" (1991, p. 43).

La acción del primer día empieza un poco antes de las dos de la tarde. Porque "Eran casi las dos cuando apareció el médico". (Los bravos, p.10), después de haber esperado un rato el viejo y el chico herido en la cantina de Manolo. Las referencias cronológicas, durante el primer día, son las siguientes:

-Eran casi las cuatro. (Los bravos, p. 11).

-[...] donde dormía la siesta [...]. (Los bravos, p. 14).

-Apareció una estrella. (Los bravos, p. 22).

-Dentro de una hora amanece. (Los bravos, p. 34).

-Eran las frías horas del alba. (Los bravos, p. 35).

El tercer día, sábado, comienza en casa de Antón, el secretario del Ayuntamiento, en la secuencia 18. Como es habitual en las novelas objetivistas: el diálogo tiene la función de informar a los lectores, la función que se desempeña el narrador omnisciente en las novelas decimonónicas. En el pasaje siguiente, a través del diálogo entre Antón y su mujer, se nos informa de que ya es de día:

- Ya me quitaste el sueño -se levantó-. Ni dormir puede un hombre.

-Si ya estabas despierto del todo ...

- ¿Qué quieres que haga ahora?

-Ya da el sol en el pueblo. (Los bravos, p. 72)

El cuarto día, el domingo, comienza con el alba "como un día cual quiera, para trabajar la madrugada" (Los bravos, p. 103). Una observación que se podría ofrecer de este día es la descripción ambiental, a modo de narración tradicional, que nos habla de las costumbres de la gente en un domingo de un pueblo campesino:

- A eso de las ocho, los que trabajaban en el campo miraron el sol que iba sobre el pueblo [...]. (Los bravos, p. 104)

-El tirador se colocaba bajo el fuego del sol [...]. (Los bravos, p. 107)

-A eso de la una, cuando el sol cae vertical sobre la tierra y los hombres pueden pisar su propia sombra [...]. (Los bravos, p. 108)

- Ya deben ser las cinco. - El médico miró su reloj, que marcaba las seis. (Los bravos, p. 110)

-Llegó el sol puesto y recogiendo las inyecciones fue a casa de don Prudencio 
[...]. (Los bravos, p. 112)

-Fuera aparecieron las primeras estrellas (Los bravos, p. 113)

-Hacía una buena noche, templada; el cielo, plagado de estrellas. (Los bravos, p. 117)

Y el quinto día, empieza con que Antonio va al otro pueblo para traer a su novia para la boda: "La mañana siguiente amaneció oscura, pero a medida que el día fue avanzando rachas de viento barriendo la niebla y las nubes altas se desvanecieron" (Los bravos, p. 118).

Otro ejemplo lo encontramos en el séptimo día, el miércoles, que se inicia con el médico sufriendo de insomnio: "Tardó en cobrar conciencia de su desvelo" (Los bravos, p. 176). Páginas más adelante, podemos apreciar que ha pasado una noche, cuando el médico sube al monte y decide quedarse allí para cuidar a un enfermo desconocido:

- ¿Va a dormir al raso?

- Sí.

- Las mañanas son muy frías.

Tardó en dormirse, pensando en Socorro, abajo, en la casa sola. (Los bravos, p. 193).

El noveno día, el viernes, se inicia con estas frases: "Los guardias subieron de madrugada. Don Prudencio los vio marchar con el viajante en medio, las manos esposadas, una hora más tarde". (Los bravos, p. 204). Y sigue pasando el tiempo: "El sol se hallaba en el vertical del cielo y el pueblo tan desierto como siempre a la hora de comer". (Los bravos, p. 208). Luego: "al anochecer se presentó en la cantina" (Los bravos, p. 211).

En este día se pueden encontrar tres puntos muy significativos en la historia: la meditación de don Prudencio ante la muerte, la decisión de Pepe de irse a la capital y la reafirmación del odio hacia el médico. Se dice que cuando uno muere contempla su pasado, y don Prudencio hace una retrospección hacia su infancia: "Según los días iban pasando, volvía diáfano a su memoria el tiempo de la infancia" (Los bravos, pp. 205206), también el médico hace lo mismo: "El médico escuchaba, y las palabras de Baltasar trajeron a su memoria un día de su infancia, cuando antes de la muerte del padre iban a pasar los veranos a Galicia" (Los bravos, pp. 116-117) 
Con la llegada de la noche se cierra el viernes y se inicia el día siguiente, el sábado, claramente con el saludo mañanero de Pepe al médico: "Buenos días" (Los bravos, p. 213). Una vez más el sol nos indica el paso del día, como aparece en estos ejemplos del texto:

Los rayos amarillentos del sol alumbraban una línea oblicua y polvorienta desde la puerta al mostrador. (Los bravos, p. 219).

-Volvieron a mediodía con el sol en lo más alto, y el aire reverberando a ras de suelo. (Los bravos, p. 220).

-Cuando el sol declinaba, a la hora de iniciarse el crepúsculo, el agua venía cálida, templada en los remansos, como una caricia para el cuerpo. (Los bravos, p. 222).

-Ambos rostros [el médico e hijo Amador] frente a frente, cercanos, se iban oscureciendo en la tarde turbia de la ventana. (Los bravos, p. 225)

De nuevo llega el domingo: "Al día siguiente fue Nuestra Señora. Amaneciendo bajaron dos pastores, con la borrega, de los puertos" (Los bravos, p. 226). He apuntado en otro domingo que hay una descripción de las costumbres del pueblo, he aquí también que se observa la costumbre de la matanza en una fecha señalada:

Unos ratos andando, otros a rastras, llegó el animal maltrecho, ciego, azul de cardenales. Lo degollaron junto a la escuela. Amador compró la pelleja, y el cuerpo sangrante quedó de un árbol, dispuesto para ser descuartizado. (Los bravos, p. 226)

El domingo ocupa la secuencia 40 y casi toda la secuencia siguiente, y en ella unos niños cuyos nombres no conoce el viejo, encuentran a don Prudencio muerto. Aun así, pasa el día como otro cualquiera, a través de un diálogo entre madre e hija:

- ¿Qué hacen ahora?

- Seguir comiendo, ¿qué quiere que hagan?

- Y tú, ¿has comido ya?

- Sí.

- ¿Por qué no comes? Te vas a poner mala.

- Con este calor no puedo. No tengo ganas. (Los bravos, p. 227)

El duodécimo día y el siguiente, lunes y martes, se resuelven en la mitad de la página 235 "El hermano llegó al día siguiente, dos horas antes del entierro" y "Al día siguiente fueron subastados, aprisa, los bienes del viejo. El médico compró la casa” (Los bravos, p. 235)

Finalmente, el último día de la novela, un miércoles, se abre así: "Un viento frío azotó el pueblo al alba. El río bajó crecido, arcilloso y las 
montañas amanecieron bajas, borradas por la niebla" (Los bravos, p. 235)

A la hora de estudiar el tiempo de Los bravos, nos llama la atención otra cosa, que, desde el primer momento, hay una imagen que presenta el tiempo detenido en el pueblo, simbolizada en el reloj de la iglesia que "aparecía inmóvil, falto de saetas, en una hora inverosímil" (Los bravos, p. 17). Esta suspensión del tiempo refleja más el aislamiento y la vida mísera de los habitantes del pueblo. Del mismo modo, señala el determinismo al que está sometida esta gente, pues, la continua repetición de la vida sin llegar a nada nuevo, hace que el tiempo sea el denominador común de los pensamientos de los habitantes del pueblo. De ahí, se siente de un tedio vital que refleja la imposibilidad de llegar a ningún progreso:

Año tras año. A la noche se acostaba maltrecha, cansada, sin saber contra qué o contra quién rebelarse, luego de moverse hora tras hora todo el día, como el asno en la noria, en torno a un provecho que no acababa de ver claro. [...] Se acabaron los juegos, comenzó la rueda. Girar, girar... ¿para qué? Su madre le hablaba desde la cama en tanto ella iba y venía con el carro, con el trillo, abonando en el caballo, sembrando para recoger como los demás hacían, sin salir de la rueda, sin apartarse ni un ápice de la voz que desde la cocina la llamaba. (Los bravos, p. 155)

Después de analizar el tiempo de la obra objeto del estudio, nos parece que predomina un tiempo progresivo y lineal, sin embargo, hay momentos de simultaneidad y algunos retrocesos, como vamos a analizar más a fondo en las páginas siguientes. Ramón Buckley intenta relacionar la simultaneidad con la novela objetivista:

La anécdota lineal es la historia en su sentido más auténtico: los acontecimientos relatados tienen un principio, un desarrollo y un final, y marcan así tres etapas bien definidas en la vida de un hombre, de varios hombres o de una comunidad. El desarrollo de la anécdota lineal produce en el lector la impresión de transcurso de tiempo real. (1973, p. 25)

\section{La simultaneidad}

La simultaneidad de las acciones, son una técnica narrativa que florece en los años cincuenta, que consiste en presentar la novela como una suma de acciones múltiples relacionadas entre sí y que ocurren al mismo tiempo, protagonizadas por personajes diferentes. Normalmente, en un espacio reducido como un pueblo o una ciudad.

En Los bravos, Jesús Fernández Santos aplica esta técnica desde las 
primeras secuencias. Ya que, en el comienzo de la tercera secuencia, se encuentra una acción incompleta: "El médico salió al huerto tras la casa, [...] vio a Pepe a la puerta del garaje, afanándose en desmontar el motor del coche" (Los bravos, p. 13). Esta escena continúa más tarde y empieza de nuevo la conversación entre los dos unas páginas después en la secuencia siguiente, cuando el médico pregunta a Pepe sobre la razón de que los habitantes del pueblo ven tan mal a don Prudencio. Mientras tanto se accede a la acción de Antón, el secretario del Ayuntamiento del pueblo, quien despierta de la siesta con su mujer y en seguida va a la casa de don Prudencio. Así, el escritor nos presenta, al mismo tiempo, dos escenas diferentes: el río donde conversan el médico y Pepe y la casa de Antón. Entonces, estamos ante dos acciones simultáneas, porque Antón se levanta de la siesta, más o menos, a las cuatro y esta es a la misma hora cuando empieza Pepe a desmontar el motor, cuando el médico le encuentra a la puerta del garaje, después de terminar de curar al chico herido, y se sabe anteriormente que el médico termina cerca de las cuatro, según la cita ya mencionada antes. Y explícitamente, tenemos un diálogo entre los tres hombres, en el que convergen las dos acciones en el mismo tiempo:

Pasando junto a la cochera de la fonda vio al médico y pensó que el anterior no tenía aquellas costumbres, al tiempo que preguntaba:

- ¿Qué, no marcha?

Los dos hombres, en mangas de camisa, le contestaron:

-i... este calor!

Y siguieron atornillando en el suelo. (Los bravos, p. 14)

La quinta secuencia, se sitúa en la casa de Amparo donde transcurre el tiempo de tarde a noche. Así, Antón, el secretario del Ayuntamiento le visita con mensaje de don Prudencio después de su siesta. La secuencia termina al caer la noche: "Fuera, sobre los campos, venía rápidamente la noche. $[\ldots]$

- Sí, sí veo; hay buena luna". (Los bravos, p. 23)

Seguimos los momentos simultáneos en la novela, desde nuestro punto de vista, podemos decir que el lunes, el día de la boda del herrero, Antonio, es uno de los mejores ejemplos representantes de la simultaneidad, ya que, este día está destacado claramente por dos grandes acciones distintas y de suma importancia en la novela; la primera, es del 
pueblo y de la iglesia donde se celebra la boda de Antonio; y la segunda es de la capital donde visita don Prudencio al médico especialista y a su hermano, que vive allí. El día empieza cuando Antonio se va a buscar a la novia al otro pueblo: "Entre dos luces salió Antonio a buscar a su novia. Era un mal camino hasta su pueblo y quería estar de vuelta antes de que el calor apretara" (Los bravos, p. 118). La boda termina con el baile a las once de la noche. Asimismo, cuando don Prudencio vuelve de Madrid y entra en su casa, sabe que Socorro se ha ido: "El reloj del comedor dio once campanas que bajaron retumbando hasta sus oídos. Pensó: "Las once, nada más que las once" (Los bravos, p. 138).

De modo paralelo, las secuencias sexta y séptima son acciones simultáneas, porque nos indican, en la séptima, la marcha de Alfredo hacia la pesca, en una noche nublada y después de la marcha del guarda desde la cantina de Manolo, quejándose de que este pueblo le toma por tonto, ya que la noche anterior alguien había puesto un cartucho en el río que había matado todas las crías de peces: "Se ocultó la luna. [...] Una ráfaga de viento trajo gotas de lluvia hasta su cara". (Los bravos, p. 29). En la sexta secuencia, ya se había mencionado la lluvia: "habrá habido tormenta en el puerto y esta noche lloverá aquí”. (Los bravos, p. 26).

Ambas secuencias se relacionan en la secuencia posterior, cuando el guardia del río tira una bala sin saber y alcanza a Alfredo. A continuación, éste es llevado a la cantina de Manolo por los hombres que pasaban cabalgando, siendo curado por el médico.

En el viernes, el segundo día de la historia, que empieza en la secuencia 10 , como ya hemos mencionado arriba y termina con la secuencia 17 , estamos ante tres momentos simultáneos; cuando el médico y el chico de Amador sufren de desvelo, aparecen representados en distintas secuencias.

[El médico] Miró el reloj: las cuatro; dentro de dos horas amanecería. Cerró los ojos y al cabo de unos minutos, cuando menos lo esperaba, un sopor frío le hizo entrar en el sueño. (Los bravos, p. 70)

[El hijo de Amador] A poco, el soplo se hizo blanco y una claridad diáfana invadió el lecho del enfermo. (Los bravos, p. 70)

Y de madrugada, de nuevo el cuerpo y el alma flotando en el vacío, hasta llegar 
a la vista y al tacto los objetos, y los pensamientos a la cabeza, sumergiéndole a su pesar en la realidad del día siguiente. (Los bravos, p .71)

Otra forma de presentar la simultaneidad, en la misma novela, es la inclusión de una descripción por el personaje que está hablando con otra persona o mientras está realizando una acción. Esto aparece de modo evidente en la conversación de Pilar con su criada, pues, encontramos una descripción, en la que Pilar ve a Pepe, a la mujer de Manolo y al otro hombre, esto nos lleva la sensación de la simultaneidad, porque la descripción de lo que ve Pilar cuando conversa con su criada:

- ¿Un tiro? Estarías soñando.

- No soñaba, no; lo que me parece es que usted duerme más de lo que dice quedó pensativa-, o no sé qué estaría haciendo, si no lo oyó.

Pilar miró por la ventana y vio a Pepe, al otro lado del río, echando agua al coche. Estaba en mangas de camisa y la mujer de Manolo le pasaba los cubos. El cielo se había vuelto color de bronce.

Al otro lado apareció Manolo y estuvo hablando unas palabras con su hermano. Pilar creyó ver a otro hombre en la puerta, pero sólo un instante, [...]. (Los bravos, p. 38).

De estas citas mencionadas arriba se muestran el uso de la técnica de la simultaneidad en Los bravos. En ésta, la simultaneidad aparece en dos formas distintas; a veces el autor nos presenta un tiempo simultáneo sin cambiar el espacio y, otras veces, el tiempo simultáneo aparece en el mismo momento, pero en lugares diferentes.

\section{La analepsis}

Aunque la mayor parte de las acciones de las obras analizadas representa una temporalidad progresiva y lineal, algunas veces Jesús Fernández Santos recurre a la analepsis cuando algunos personajes recuerdan su pasado. Según Villanueva quien dice que:

En efecto, siempre que hay una dislocación entre el orden de la historia y el del discurso surge -el término lo aceptaremos de Genette- una anacronía, que puede ser hacia atrás desde la línea temporal predomínate o relato primario analepsis o flash back en la jerga cinematográfica- o hacia adelante- prolepsis o flash forward.(1989, p. 48)

En Los bravos, tenemos un párrafo que merece la pena ser analizado por su exposición complicada, pues, en él aparece una anacrónica; analepsis interna y externa. En la interna Alfredo le pregunta al médico si se quedaría en el pueblo; en la externa el médico recuerda su tiempo de 
ayudante:

Era otro día. De nuevo vino el frío; se estremeció metiendo las manos en los bolsillos. Alfredo le había preguntado si se quedaría o si pensaba marchar como el otro. ¿Por qué se había marchado el otro? Era evidente: la buena vida. Pensó en sus compañeros ayudando en las consultas; con el tiempo, la clientela de sus patrones pasaría a ellos, podrían casar con sus hijas para perpetuar la estirpe, la buena raza. [...] Algunos quedarían en modestas consultas, según su habilidad o su suerte, o en seguros, o en sociedades, pero, ¿quién pensaba en meterse en un pueblo? (Los bravos, p. 179).

El tiempo principal de la acción es durante el viaje del médico al monte para curar un pastor enfermo de pulmonía. La pregunta de Alfredo es una anacronía cuyo alcance total se mantiene en el interior del relato primario (analepsis interna) y el recuerdo del médico cuya amplitud se mantiene fuera del relato primario (analepsis externa), y vienen marcados con el pretérito imperfecto (pensaba, preguntaba) frente al indefinido del tiempo principal (vino, se estremeció, tropezó).

\section{La prolepsis}

En relación al tratamiento temporal del corpus, se halla también el uso de la anticipación, una técnica que consiste en mencionar un asunto o un personaje, con una breve referencia, y omitir su alusión durante varias páginas para recuperarlo más adelante detalladamente. Se considera que, gracias al empleo de esta técnica cinematográfica, Los bravos, llama la atención del público poderosamente, según Gil Casado: "El interés del lector se mantiene no por el procedimiento de no revelar una situación o un personaje sino dejando que se vayan mostrando lentamente por sí mismos" (1975, p. 249).

En el mismo sentido, S. Pastor opina que esta alternancia de las acciones, aumenta la intriga, y llama la atención de los lectores, según sus palabras:

Todo ello acrecienta la intriga y mantiene el interés del lector al no revelársele una situación concreta o la intencionalidad de un personaje, sino dejando que se manifieste por sí solo; la narración procede, por tanto, de lo ambiguo a lo concreto. (1994, p. 240)

El uso de esta técnica cinematográfica del anticipo, se presenta tanto en la primera novela, Los bravos, como en la obra posterior del autor. Según D. Santos, en Los bravos existen tres mecanismos de técnicas narrativas 
que le dan un aire particular a la hora de describir: el uso de técnicas cinematográficas, anticipación y ambiguiedad. D. Santos dice:

Sobre los recursos de la anticipación y ambigüedad descriptiva, es normal a lo largo de la novela el dar detalles, hacer descripciones de personas o cosas que no aparecen hasta varias líneas más tarde en la página siguiente. Este mecanismo contribuye a dar unidad a la descripción, al mismo tiempo que crea una intriga en el lector. (2001, p. 72).

Gil Casado muestra como ejemplo de esta técnica, la enfermedad de don Prudencio; pues, primero, aludida de forma somera en el diálogo de los vecinos y más tarde se desarrolla durante el viaje de don Prudencio a la capital para consultar a un especialista (1975, pp. 247-248).

Otro ejemplo lo encontramos en la presentación del personaje del estafador. Así, al principio, nos describe cómo va vestido: "vestía una chaqueta negra y un viejo y gastado pantalón, pero lo que más llamó su atención fueron los zapatos negros". (Los bravos: 44), luego, aparece el río donde se encuentra Antón. A continuación, reaparece otra vez con más detalles: "Ahora estaba junto a él, y podía examinarle de cerca a su gusto. Una cara carnosa, enrojecida por el sol, cribada de profundos poros, sobre la que los aros de alambre de unos lentes dejaban marcada una huella cruda, blanquecina" (Los bravos, pp. 44-45).

Más tarde, se menciona la razón de su presencia en el pueblo que aparece en su conversación con Amparo, tratando de convencer los habitantes del pueblo para confiarle y darle sus ahorros:

A eso de las cuatro, cuando todo el mundo duerme la siesta, el forastero empezó a charlar con Amparo. [...] y sacó de su cartera un fajo de papeles impresos.

-Usted nos da su dinero y nosotros se lo guardamos.

-No, si yo ya sé dónde tengo que guardarlo... (Los bravos, pp. 55-56).

En el anterior ejemplo aparece la influencia de la técnica cinematográfica que según S. Pastor "se basa en avanzar en planos distintos, de lo abstracto a lo concreto, hasta enfocar el elemento que interesa resaltar". (1994, p. 695).

\section{Conclusiones}

Al final, podemos concluir que Los bravos es una historia lineal, con 
reducción temporal y espacial, una de las características de la novela del medio siglo, con breves retrospecciones, cuando los personajes recuerdan un momento en su pasado.

También, se observan la inclusión de la técnica de la simultaneidad, utilizada para dar una mayor impresión de la realidad cotidiana. Pues, la vida de cada persona en el pueblo es una parte del mundo en el que suceden acciones simultáneas. Así, Jesús Fernández Santos no realiza sólo una exposición paralela de secuencias, sino que introduce éstas en pleno diálogo para darnos información sobre lo que ocurre en otro lugar o para enfocar otra acción, que sucede en el mismo momento.

\section{Bibliografía}

Bobes Naves, M. (1998). La novela. Editorial Síntesis.

Buckley, R. (1973). Problemas formales en la novela española contemporánea. Ediciones Península.

Chatman, S. (1990). Historia y discurso: la estructura narrativa en la novela y en el cine. Taurus.

Diego Santos, J. (2001). Léxico y sociedad en Los bravos de Jesús Fernández Santos. Universidad de Alicante.

García de Nora, E. (1937). La novela española contemporánea (1939- 1967). vol. III. Gredos.

Gil Casado, P. (1975). La novela social española (192 -1971). Editorial Seix Barral.

Jiménez Madrid, R. (1991). El universo narrativo de Jesús Fernández Santos (19541987). Universidad de Murcia.

Pastor Cesteros, S. (1994). La obra narrativa de Jesús Fernández Santos y su relación con el cine. Universidad de Alicante.

Plata Tasende, A. M. (2000). Diccionario de términos literarios. Espasa Calpe.

Sanz Villanueva, S. (1980). Historia de la novela social (1942-1975). vol. I. Alhambra.

Sobejano, G. (1975). Novela española de nuestro tiempo (en busca del pueblo perdido). Segunda edición. Prensa Española.

Villanueva, D. (1989). El comentario de textos narrativos: La novela. Ediciones Júcar. 